\title{
Fermentation process optimization and chemical composition analysis on black tea wine
}

\author{
Chun Zou ${ }^{1}$, Yongquan $\mathrm{Xu}^{1}$, Jianxin Chen ${ }^{1}$, Ruyi $\mathrm{Li}^{1}$, Fang Wang ${ }^{1}$, and Junfeng Yin ${ }^{1}$ * \\ ${ }^{1}$ Tea Research Institute Chinese Academy of Agricultural Sciences, 310008 Hangzhou China
}

\begin{abstract}
In this study, the fermentation process of black tea wine was optimized, and the changes in catechins, organic acids, and aroma components during fermentation were investigated. The optimal fermentation conditions for tea wine were determined as follows: the addition of $15 \%$ sucrose and $0.75 \%$ tea leaves, and a fermentation temperature of $25{ }^{\circ} \mathrm{C}$. Under the optimal conditions, the alcohol content and sensory evaluation score of tea wine were $8.9 \% \mathrm{ABV}$ and 88 , respectively. The contents of catechins and organic acids in tea wine were $21.29 \mathrm{mg} / \mathrm{L}$ and $3.68 \mathrm{mg} / \mathrm{mL}$, which were 1.32 -fold and 10.51 -fold higher than those of tea infusion, respectively. L-malic acid was the main organic acid in tea wine, which accounted for $56.3 \%$ of the total amount of organic acids. A total number of 34 and 33 kinds of aroma components were detected in tea wine and tea infusion, respectively. The main aroma components of tea wine were esters and alcohols, which accounted for $39.7 \%$ and $45.9 \%$ of the total amount of aroma components, respectively, while the main aroma components of tea infusion were esters and aldehydes, accounting for $34.6 \%$ and $33.2 \%$, respectively.
\end{abstract}

\section{Introduction}

Tea wine combines the advantages of tea and wine, which has been consumed for many years ${ }^{[1,2]}$. There are many active compounds found in tea wine, such as catechins, organic acids, caffeine, and alcohols ${ }^{[3,4]}$. These active compounds provide tea wine with potential benefits for human health, including antioxidant, antiaging efficacy, and immunity improvement ${ }^{[5-7]}$.

Fermented tea wine is made by alcoholic yeast fermentation of tea and other raw material. Due to the lack of sugar in tea leaves, carbon source should be supplemented in fermentation, such as sucrose, rice, sorghum, and fruit ${ }^{[8-10]}$. Therefore, it is important for tea wine to optimize the concentration of the raw materials. Moreover, the quality of tea wine is influenced by the controlled fermentation conditions ${ }^{[11]}$.

In this study, Keemun black tea and sucrose were used as raw materials in the fermentation of tea wine. The effects of sucrose and tea concentration, and fermentation temperature on the alcohol content and sensory evaluation score of tea wine were investigated to find the optimal fermentation conditions. In order to identify the various chemical components of tea wine, the contents of catechins and organic acids were measured using high performance liquid chromatography (HPLC), and the aroma components were detected using Headspace Solid Phase Microextraction (HS-SPME) and Gas ChromatographyMass Spectrometry (GC-MS). The research results will provide a scientific basis for developing the tea wine industry.

\section{Materials and methods}

\subsection{Reagents and materials}

Black tea (Keemun black tea) was purchased from Tianzhihong Co. (Huangshan, China). Sucrose was purchased from local market in Hangzhou, China. Saccharomyces cerevisiae was purchased from Angel Yeast Co., Ltd. (Yichang, China).

Organic acids (acetic, gluconic, malic, lactic, succinic, citric, and gallic acid), and catechins ((-)epigallocatechin gallate (EGCG), (+)-catechin (C), (-)catechin gallate $(\mathrm{CG}),(-)$-epicatechin gallate $(\mathrm{ECG}),(-)-$ gallocatechin gallate (GCG)) standards were purchased from SigmaAldrich Shanghai Trading Co., Ltd. (Shanghai, China). Methanol and acetonitrile of high performance liquid chromatograph (HPLC) grade were purchased from Merck Co. (Darmstadt, Germany). All other chemicals were of analytical grade and purchased from Sinopharm Chemical Reagent Co., Ltd. (Shanghai, China).

\subsection{Fermentation process optimization of tea wine}

The sugared tea infusion was prepared as the following steps: different concentrations $(5,7.5,10,15,20$, or $30 \%)$ of sucrose solution were sterilized at $121^{\circ} \mathrm{C}$ for $15 \mathrm{~min}$. Then, different concentrations $(0.5,0.75,1$, or $1.25 \%)$ of tea leaves were infused at $90{ }^{\circ} \mathrm{C}$ for $10 \mathrm{~min}$. The tea

\footnotetext{
*Corresponding author: yinjf@tricaas.com
} 
leaves were removed and the tea infusion was transferred into a sterilized glass jar, which was cooled down to room temperature before inoculation.

The sugared tea infusion was inoculated with $0.05 \%$ of $S$. cerevisiae, which had been activated. Fermentation was carried out at different temperature $\left(20,25\right.$, or $\left.30^{\circ} \mathrm{C}\right)$ for $12-14 \mathrm{~d}$.

\subsection{Sensory analysis}

The tea wine was scored by a trained team of 8 panelists (4 men and 4 women, 23-50 years old) from the Tea Research Institute of the Chinese Academy of Agricultural Sciences. Scores for taste, flavor, and appearance were given by each member of the team. The criteria for sensory evaluation were referred to "Handbook of Wine Industry" [12].

\subsection{Determination of organic acids and catechins}

Organic acids were analyzed using HPLC equipped with a UV-DAD detector set at $220 \mathrm{~nm}{ }^{[13]}$. Catechins were analyzed using HPLC equipped with a UV-DAD detector set at $280 \mathrm{~nm}^{[14]}$.

\subsection{Analysis of aroma components}

HS-SPME-GC-MS method was used to determine volatile aromas in tea wine ${ }^{[15]}$.

\subsection{Statistical analysis}

All results were presented as mean \pm standard deviation (SD) of three replicates. The level of statistical significance among the means was analyzed by one-way ANOVA using SPSS (version 18.0; SPSS Inc., Chicago, IL).

\section{Results and discussion}

\subsection{Effect of sucrose addition on properties of tea wine}

Sucrose was added as the carbon source for the fermentation of tea wine. The effect of sucrose addition on properties of tea wine was investigated. As shown in Fig. 1, when 5-15\% sucrose was added, the alcohol content of tea wine increased remarkably as the sucrose concentration increased. But when the sucrose concentration was higher than $15 \%$, the alcohol content of tea wine changed little. The high sucrose concentration could lead to high osmotic pressure in tea broth, which would inhibit the metabolic activity of yeast ${ }^{[16]}$. Moreover, when the sucrose concentration was $15 \%$, the tea wine reached the maximum sensory evaluation score, which was 83 . Therefore, the optimal sucrose concentration was $15 \%$.

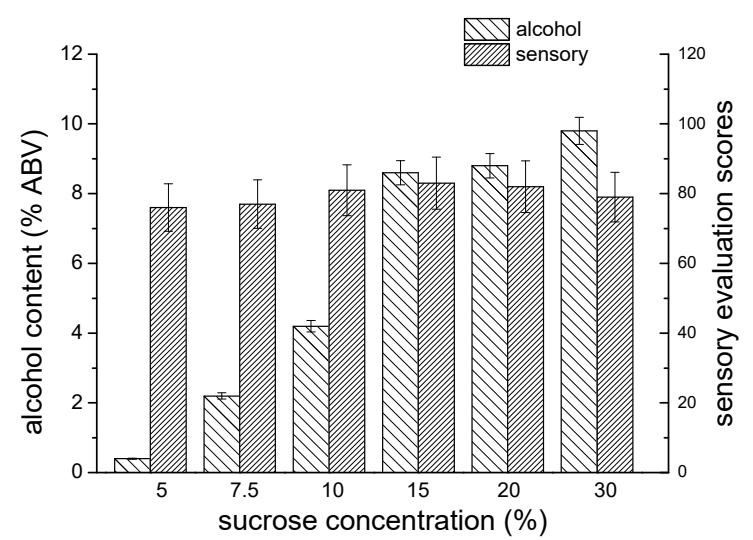

Fig. 1. Effect of sucrose addition on alcohol content and sensory evaluation score of tea wine.

\subsection{Effect of tea addition on properties of tea wine}

Tea infusion not only provided nutrients for yeast, but also influenced the sensory properties of tea wine ${ }^{[17]}$. As shown in Fig. 2, as the tea concentration increased, the alcohol content of tea wine increased at first, and finally tended toward stable. However, when the tea concentration was higher than $1 \%$, the sensory evaluation score of tea wine decreased significantly. The high tea concentration could increase the bitterness and astringency of tea wine. When the optimal concentration of tea $(0.75 \%)$ was supplied, the alcohol content and sensory evaluation score of tea wine were $8.6 \% \mathrm{ABV}$ and 84 , respectively.

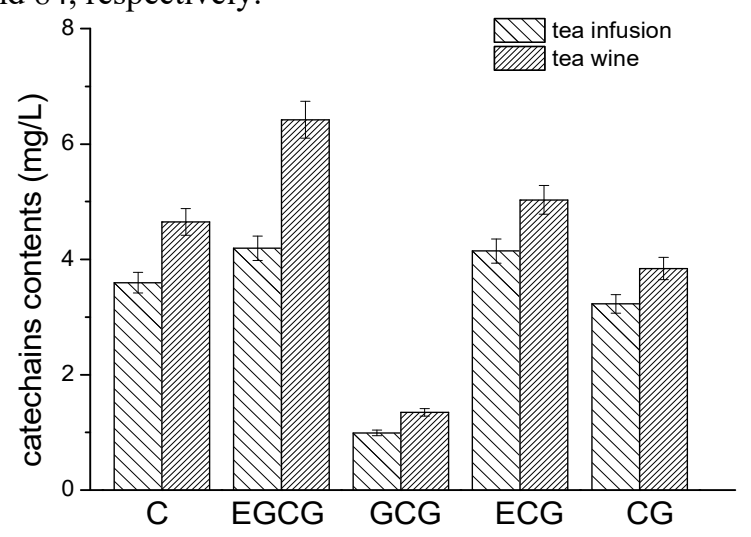

Fig. 2. Effect of tea addition on alcohol content and sensory evaluation score of tea wine.

\subsection{Effect of temperature on properties of tea wine}

Temperature influences the growth and metabolism of yeast. As shown in Fig. 3, the difference of alcohol contents of tea wine fermented under different temperature conditions $\left(20,25\right.$, and $\left.30^{\circ} \mathrm{C}\right)$ were insignificant. On the other side, the sensory evaluation score of tea wine fermented under $25^{\circ} \mathrm{C}$ was higher than those under 20 and $30{ }^{\circ} \mathrm{C}$. Therefore, the optimal temperature was $25^{\circ} \mathrm{C}$. Under the optimal temperature condition, the alcohol content and sensory evaluation score of tea wine were $8.9 \% \mathrm{ABV}$ and 88 , respectively. 


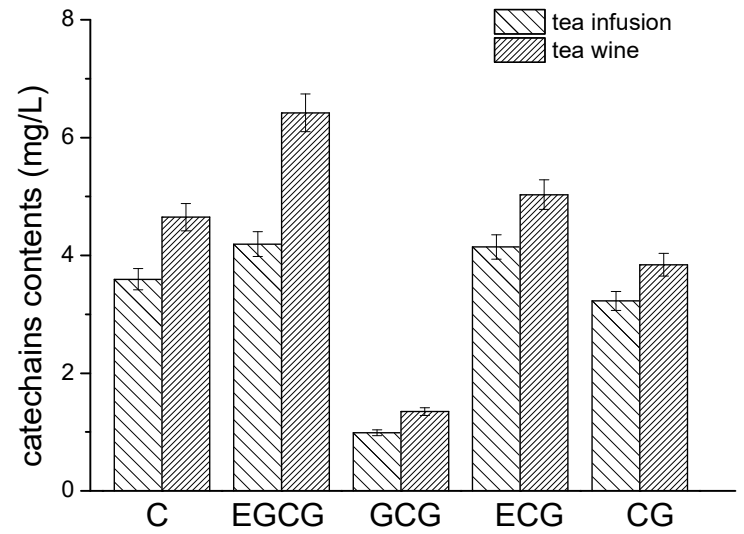

Fig. 3. Effect of temperature on alcohol content and sensory evaluation score of tea wine.

\subsection{Analysis of functional components}

According to the abovementioned fermentation process optimization, the addition of $15 \%$ sucrose and $0.75 \%$ tea leaves, and fermentation temperature of $25{ }^{\circ} \mathrm{C}$ were indicated as the optimal fermentation conditions. The functional components including catechins and organic acids in tea infusion and tea wine under the optimal fermentation conditions were detected. As shown in Fig. 4 , the contents of 5 catechins in tea wine were all higher than those of tea infusion. The total content of catechin in tea wine was $21.29 \mathrm{mg} / \mathrm{L}$, which was 1.32 -fold higher than that of tea infusion. Zhao et al ${ }^{[18]}$ reported the similar changes of catechins during the tea wine fermentation.

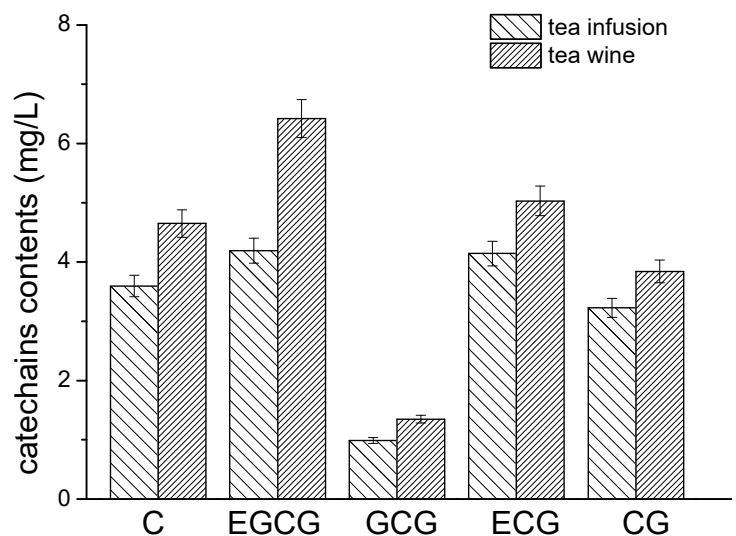

Fig. 4. The catechins contents of tea infusion and tea wine.

As shown in Fig. 5, all organic acids detected in tea wine except gallic acid accumulated during the fermentation, while the concentration of gallic acid did not change. The total content of organic acid in tea wine was $3.68 \mathrm{mg} / \mathrm{mL}$, which was 10.51 -fold higher than that of tea infusion. L-malic acid was the main organic acid in tea wine, which accounted for $56.3 \%$ of the total organic acids.

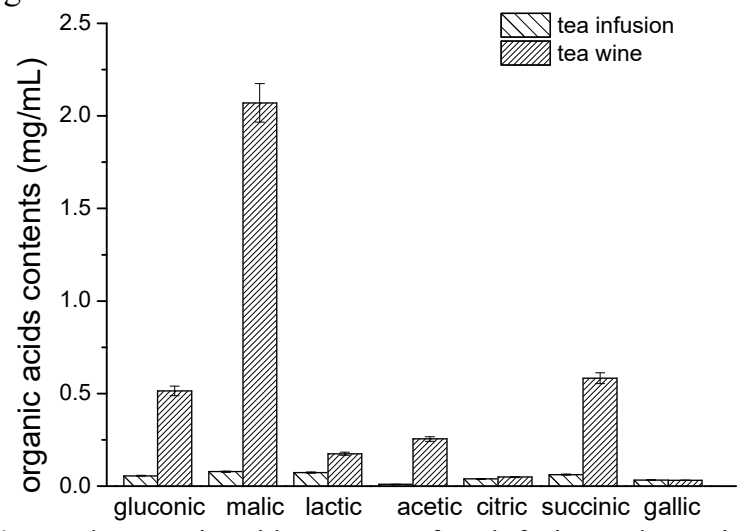

Fig. 5. The organic acids contents of tea infusion and tea wine.

\subsection{Analysis of aroma components}

As shown in Table 1, there were 33 and 34 aroma components detected in tea infusion and tea wine, respectively. Tea infusion contained 5 esters, 4 alcohols, 8 alkanes, 6 aldehydes, 5 alkenes, 3 ketones, 1 phenols and 1 ethers. After the yeast fermentation, tea wine contained 13 esters, 4 alcohols, 7 alkanes, 3 aldehydes, 1 alkenes, 2 ketones, 3 phenols and 1 ethers. The main aroma components of tea wine were esters and alcohols, which accounted for $39.7 \%$ and $45.9 \%$ of the total amount of aroma components, respectively, while the main aroma components of tea infusion were esters and aldehydes, accounting for $34.6 \%$ and $33.2 \%$, respectively.

Esters play an important role in the pleasant and mellow wine aroma, and improve the odor of the wine to be more harmonious and balanced ${ }^{[19]}$. There were 8 esters generated in tea wine; ethyl sorbate was the most abundant ester component, with the content of 54.04 $\mu \mathrm{g} / \mathrm{L}$. In addition, 2 esters contents increased and other 3 esters contents decreased after the fermentation.

Alcohols are also important aroma components in wine ${ }^{[20]}$. Isoamyl alcohol and phenylethyl alcohol were the main alcohols generated in tea wine, with the content of 44.07 and $91.09 \mu \mathrm{g} / \mathrm{L}$, respectively. However, there were 2 alcohols (2-ethyl hexanol and linalool) disappeared after the fermentation. Moreover, there were 2 phenols, 1 ethers and 1 alkenes generated in tea wine, but 5 alkenes, 1 alkanes, 3 aldehydes, 1 ethers and 1 ketones were disappeared after the fermentation.

Table 1. Contents of aroma components in tea infusion and tea wine.

\begin{tabular}{cccc}
\hline \multirow{2}{*}{ type } & name & \multicolumn{2}{c}{ contents $(\mu \mathrm{g} / \mathrm{L})$} \\
\cline { 3 - 4 } & & tea infusion & tea wine \\
\hline \multirow{3}{*}{ esters } & lactic acid ethyl ester & 0 & 4.94 \\
& isoamyl acetate & 0 & 1.68 \\
& ethyl caproate & 0 & 7.17 \\
& ethyl sorbate & 0 & 54.04 \\
\hline
\end{tabular}




\begin{tabular}{|c|c|c|c|}
\hline & phenethyl acetate & 0 & 2.16 \\
\hline & ethyl caprylate & 0 & 21.06 \\
\hline & monoethyl succinate & 0 & 12.35 \\
\hline & diethyl succinate & 0 & 5.17 \\
\hline & ethyl dodeconoate & 0.86 & 1.96 \\
\hline & methyl salicylate & 1.29 & 5.22 \\
\hline & 2-propenoic acid, butyl ester & 45.72 & 6.51 \\
\hline & butyl butyrate & 1.79 & 0.64 \\
\hline & (Z)-3-Hexenyl hexanoate & 1.50 & 0.49 \\
\hline \multirow{6}{*}{ alcohols } & isoamyl alcohol & 0 & 44.07 \\
\hline & phenylethyl alcohol & 0 & 91.09 \\
\hline & trans-nerolidol & 2.04 & 4.39 \\
\hline & geraniol & 4.36 & 3.13 \\
\hline & 2-ethyl hexanol & 2.43 & 0 \\
\hline & linalool & 3.72 & 0 \\
\hline \multirow{8}{*}{ alkanes } & n-dodecane & 0.84 & 5.87 \\
\hline & n-tetradecane & 2.75 & 7.82 \\
\hline & n-pentadecane & 1.78 & 1.83 \\
\hline & n-hexadecane & 2.59 & 4.53 \\
\hline & n-heptadecane & 0.92 & 1.23 \\
\hline & n-octadecane & 0.26 & 1.15 \\
\hline & 5-ethyl-2-methyl-octane & 1.26 & 1.55 \\
\hline & 2,2,4,6,6-pentamethylheptane & 2.63 & 0 \\
\hline \multirow{6}{*}{ aldehydes } & 5-hydroxymethylfurfural & 34.07 & 5.18 \\
\hline & benzaldehyde & 1.03 & 0.47 \\
\hline & decanal & 1.36 & 1.30 \\
\hline & nonanal & 9.14 & 0 \\
\hline & safranal & 1.94 & 0 \\
\hline & 2,5-Furandicarboxaldehyde & 1.46 & 0 \\
\hline \multirow{3}{*}{ phenols } & eugenol & 0 & 2.37 \\
\hline & 2,4-di-t-butyl-6-nitro- phenol & 0 & 1.32 \\
\hline & 2,6-di-tert-butyl-phenol & 2.34 & 2.97 \\
\hline \multirow{2}{*}{ ethers } & ethyl geranyl ether & 0 & 3.61 \\
\hline & n-butyl ether & 6.18 & 0 \\
\hline \multirow{6}{*}{ alkenes } & terpinolene & 0 & 1.78 \\
\hline & ocimene & 2.00 & 0 \\
\hline & beta-thujene & 2.83 & 0 \\
\hline & beta-caryophyllene & 1.35 & 0 \\
\hline & (Z)-beta-farnesene & 1.31 & 0 \\
\hline & alph-farnesene & 1.52 & 0 \\
\hline \multirow{3}{*}{ ketones } & damascenone & 0.66 & 1.21 \\
\hline & 3,4-dehydro- $\beta$-ionone & 1.24 & 0 \\
\hline & beta-ionone & 2.64 & 0.39 \\
\hline
\end{tabular}

\section{Conclusions}

In this study, the optimal fermentation conditions for tea wine were determined as follows: the addition of $15 \%$ sucrose and $0.75 \%$ tea leaves, and a fermentation temperature of $25{ }^{\circ} \mathrm{C}$. The contents of catechins and organic acids in tea wine were $21.29 \mathrm{mg} / \mathrm{L}$ and 3.68 $\mathrm{mg} / \mathrm{mL}$, which were 1.32-fold and 10.51-fold higher than those of tea infusion, respectively. L-malic acid was the main organic acids in tea wine, which accounted for $56.3 \%$ of the total amount of organic acids.

A total number of 34 kinds of aroma components existed in tea wine, including 13 esters, 4 alcohols, 7 alkanes, 3 aldehydes, 1 alkenes, 2 ketones, 3 phenols and 1 ethers, while a total number of 33 kinds of aroma 
components existed in tea infusion, including 5 esters, 4 alcohols, 8 alkanes, 6 aldehydes, 5 alkenes, 3 ketones, 1 phenols and 1 ethers. The main aroma components of tea wine were esters and alcohols, which accounted for $39.7 \%$ and $45.9 \%$ of the total amount of aroma components, respectively, while the main aroma components of tea infusion were esters and aldehydes, accounting for $34.6 \%$ and $33.2 \%$, respectively.

\section{Acknowledgments}

This research was supported by the Central Publicinterest Scientific Institution Basal Research Fund (No. 1610212017012) and Zhejiang Provincial Key Research and Development Project (2019C02072).

\section{References}

1. Y. Xu, H. He, X. Pan, J. Lin, H. Feng. J. Tea, 4602 (2020)

2. S. O. Aroyeun, O. Olubamiwa, M. A. K. Ogunjobi. Brit. Food J., 1071 (2005)

3. Y. Li, S. Zhang, Y. Sun. Sau. J. Biol. Sci., 271 (2020)

4. Y. Li, Y. Lin, L. Yao, Y. Sun. T. Food Ind., 3907 (2018)

5. J. Wang, H.Yang, Y. Zang, X. Wu, Z. Li, Z. Xu. Food Ferment. Ind., 4406 (2018)

6. Y. Li, Y. Sun. China Food Addit., 3002 (2019)

7. G. Levan, T. Tamar, J. Merab, G. Manana. Brazil. J. Food Tech., 22 (2019)

8. V. K. Joshi,V. Kumar. J. I. Brew., 1232 (2017)

9. J. Lin, X. Liu, S. Tian, Q. He, Z. Zhang, C. Shen. T. Food Ind., 4007 (2019)

10. C. Zou, H. Zhou, S. Chen. China Brew., 3602 (2017)

11. R. Wang, X. Jiang, L. Chen, J. Zhu. Food Res. Dev., 4015 (2019)

12. B. Zhu, R. Dai, G. Zhao. Handbook of wine industry (China Lig. Ind. P., 1995)

13. C. Tu, S. Tang, F. Azi, W. Hu, M. Dong. J. Func. Foods, 52 (2019)

14. Q. Cao, C. Zou, Y. Zhang, Q. Du, J. Yin, J. Shi, S. Xue, Y. Xu. Food Chem., 277 (2019)

15. G. Liu, J. Sun, X. He, Y. Tang, J. Li, D. Ling, C. Li, L. Li, F. Zheng, J. Sheng, P. Wei, M. Xin. Food Chem., 256 (2018)

16. H. Li, C. Shang, X. Shen, T. Feng, C. Niu, Y. Wu. Food Sci. Tech., 4102 (2016)

17. T. He, F. Liu, L. Du, D. Xiao. Liq. Mak. Sci. Tech., 10 (2015)

18. X. Zhao, H. Xu, R. Yang. Food Sci. 3505 (2014)

19. G. Duan, Y. Liu, H. Lv, F. Wu, R. Wang. Biotechnol. Biotec. Eq., 341 (2020)
20. M. Martínez-Pérez, A. Bautista-Ortín, P. PérezPorras, R. Jurado, E. Gómez-Plaza. Foods, 96 (2020) 